\title{
Research on the Multicast Trickle Algorithm Based on Redundant Touting Technique \\ Rong Nie, Yi-Fa MENG
}

Beijing Engineering Research Center of Security Alarm and Inspection,Beijing, China

Keywords:RPL; the multicast trickle algorithm; redundancy tree routing

\begin{abstract}
In the power information collection networking, the design of the routing protocol has a great impact on network performance. This paper proposes increase routing redundancy tree in the basis of low-power lossy network RPL routing protocols, and study the multicast trickle algorithm and complete the simulation of the algorithm.
\end{abstract}

\section{Introduction}

In recent years, the smart grid has become a trend. Electricity Information collection system is the basic application system of the intelligent electricity power service system. The electricity Information collection system can ensure the safe operation of power, and play a major role on the orderly use of electricity. Electricity Information collection system mainly use flooding metering mode. The master can't collect the electricity information of some fixed meters, such as electricity meter data, abnormal event information, remote software upgrade, program updates and so on.

Therefore, we proposed multicast trickle algorithm of the adding redundant tree routing based on RPL routing protocol[1-3]. And we complete the construction of 29-node system. Finally, the multicast algorithm of RPL multicast routing protocol is applied to the meter reading to improve the insufficient of the broadcast meter reading. And we achieve end to end control.

\section{Increasing redundancy tree routing}

In the access network course of the tree router, if there are two or more routers can be selected as a parent node, the node can select the optimal router node as a parent node and select a redundant router as a backup. Whenthe link of the node and parent node is broken, it can be quickly build temporary routing and route repair by the redundant routing. This can maintain the feature of constant node address.

\section{A. The selection policy of redundancy router}

These are the reasonable alternative method of the redundant routers: Preferentially choose the sibling node of the parent node as a redundant router. Second, choose the routing which has the smallest number of hops to the parent node router as a redundant router.

As shown in Fig.1, the dotted line is a redundant route connection. When the communication link of a node and parent node is failed, the node can establish a connection with the network through redundant router.

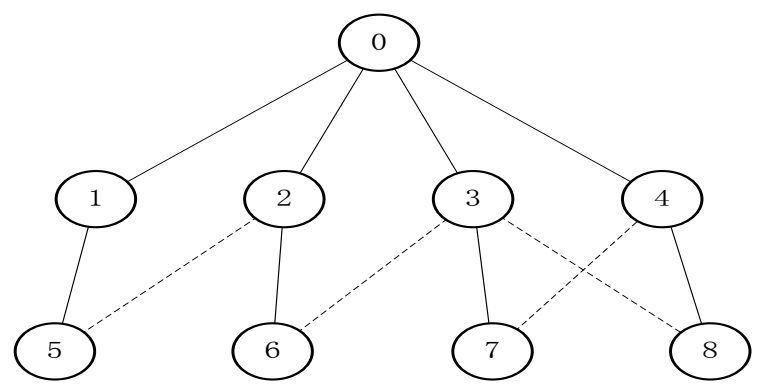

Fig. 1. The topology of the tree redundant routing 


\section{B. Fast temporary routing}

When the link between a node and parent node is failed, the node can quickly realize temporary routing. And the node chooses the better next hop to transponder data. If a node has a redundant router, the data can be submitted directly to the redundant router; if the node hasn't a redundant router, the node can choose the smallest depth node from the neighbor table for forwarding data.

As shown in Fig.2, the solid line is the original tree routing. When the node 4 failed, the node 8 forwards the data to its redundant router 3 , as shown by the dashed line.

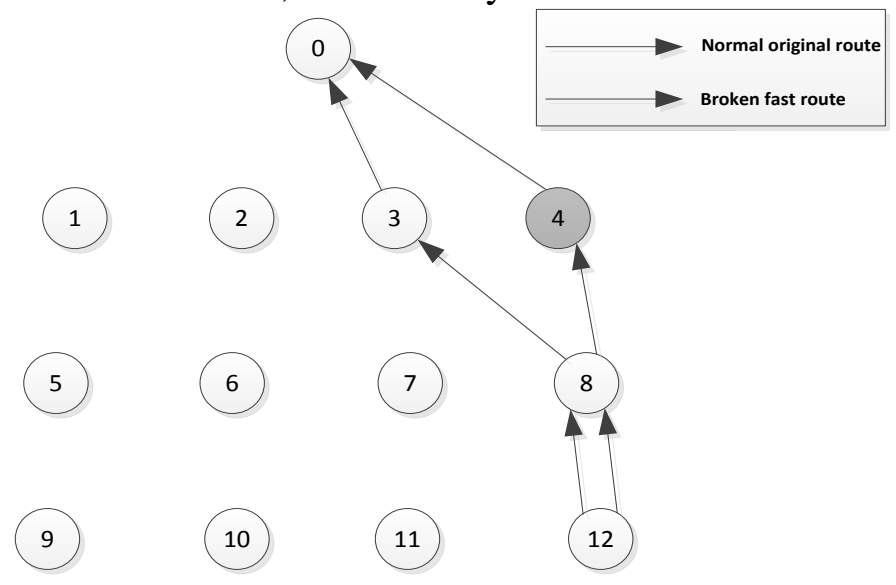

Fig. 2. Uplink fast routing schematic

\section{Tree routing repair}

After starting the fast temporary route, if the original tree link is recovered within a certain time, the rapid temporary route is canceled. The node works according to the original tree routing. The disconnected node over a certain time is still failed. Then the child node of the disconnected of nodes can repair the routing. If the redundant routing nodes are the sibling node of the disconnected node, we can directly select the redundant router as a proxy parent node; if the redundant routing is not the sibling node of the broken node, we can re-find the optimal redundant nodes in the neighbor table as a proxy parent node. The lookup process is consistent withthe choosing process of the redundant router. The route repair method is shown in Fig. 3.

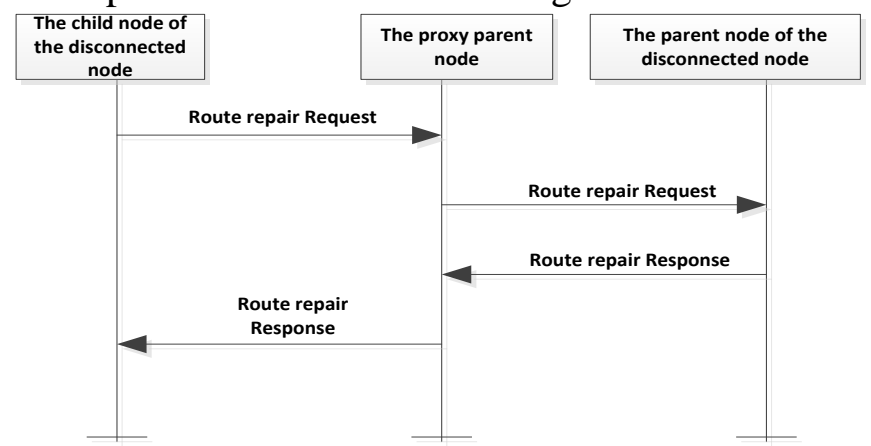

Fig. 3. The Interactive process of route repair

\section{Micro-power wireless communication RPL multicast routing}

When creating DODAG, RPL use trickle timer to determine the time of the multicast trickle timer. When the DODAG network is stable, we reduce the sending of RPL message. When detecting the inconsistencies of DODAG, we accelerate to send RPL messages. And reduce the spending of the control layer through the trickle timer.

In the multicast trickle algorithm, we control the transmission rate by using adaptive mechanism, so that in any case able the node can hear enough package to maintain consistency. Furthermore, the 
states of trickle algorithm are less. It only depends on the local to complete communication. we can guarantee routing information synchronization by controlling the transmission rate and monitoring a small amount of packet. Therefore, multicast trickle algorithm can adapt to the no connection, lossy and low-power network status. The network does not need the complex code and status[4-5].

The trickle behavior is controlled by a number of parameters.

$\mathrm{T}$ : time value; $\mathrm{T}$ is between the interval [I, I / 2].

C: redundancy counter.

I: the length of current communication interval.

$\mathrm{K}$ : redundant constants (obtained from DODAG root).

$\mathrm{I}_{\mathrm{min}}$ : we get the minimum value I by DIO. $\mathrm{I}_{\min }=2^{\text {DIoIntervalMin }} \mathrm{ms}$, DIOIntervalMin is notice by the message DIO of DODAG root.

$I_{\text {doubling }}: I_{\text {doubling }}$ is DIOIntervalDoubling. It will be noticed by DODAG roots in the DIO

$I_{\max }$ : the maximum value $I_{\max }=I_{\min } \times 2 I_{\text {doubling. }}$.

In RPL trickle algorithm, the node set Idouble and Imin according to the original value from DIO, $\mathrm{I}=\mathrm{Imin}, \mathrm{C}=0, \mathrm{~T}$ is a random value between the interval [I, I / 2].

When the node receive consistent DIO message from its DODAG parent node, C counter will progressively increase. When the timer expires, $\mathrm{C}$ counter decides whether needing one more DIO multicast message by comparing with the constant of RPL. However, I will double due to the expiring of communication interval. At this moment, reset the counters $\mathrm{C}$ and assign a new value for $\mathrm{T}$, until I reach the maximum Imax.

The specific steps of trickle algorithm are shown in Fig. 4.

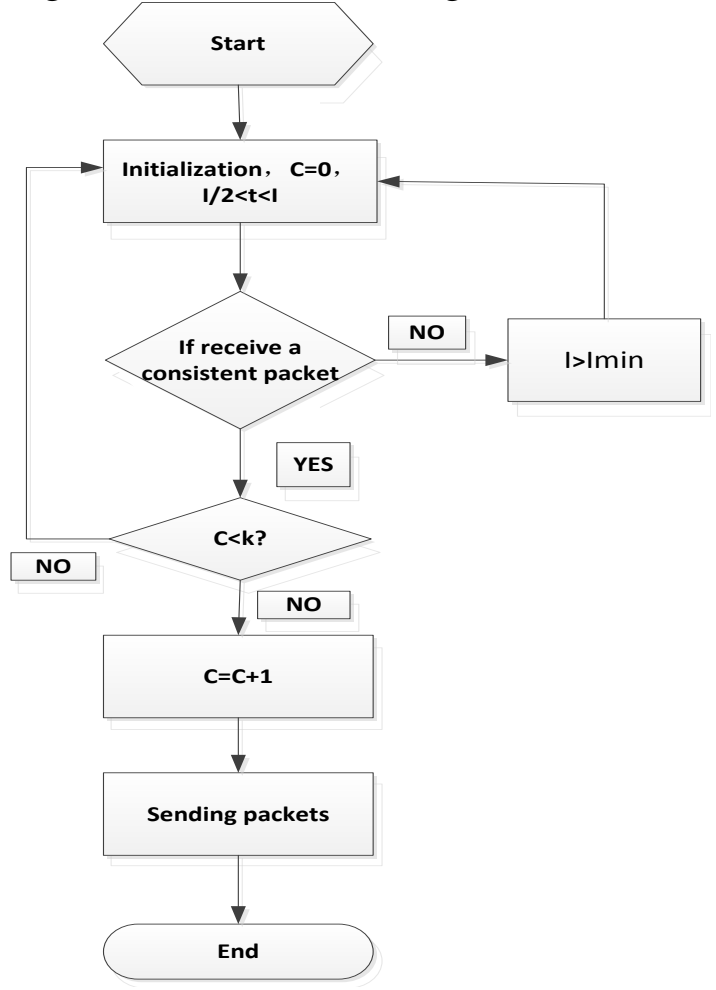

Fig. 4. The steps of trickle algorithm

\section{Multicast routing algorithm Simulation}

This article selects InstantContiki2.7 as the simulation platform and selects The Contiki Network Simulator: COOJA as a network simulation tools [7-8]. The sensing region of simulation is $100 \mathrm{~m}^{*} 100 \mathrm{~m}$. There are a gateway node, two routing nodes and 26 ordinary nodes in the simulation environment. The area of the nodes power is $\mathrm{Rtx}=75 \mathrm{~m}$; we set node manually distributed in the sensing region. The nodes select Radio Environment (UDGM) according to the way to increase the 
redundant tree routing. The platform of node is sky. Set the time initial interval of generated DIO as $1 \mathrm{~s}$, and the time interval varies with the simulation time.

Master support control command, maintenance, and the sending down of upgrade program [4-5]. And support the partial measurement of power data. When the master sends commands to the power meter, the specific multicast address package is delivered to all interfaces identified by multicast address[6-8]. The requested node multicast address is assigned to the interface. Thereby reduce traffic congestion and save network resources. IPv6 uses multicast listener discovery (MLD) in the management of multicast group.

The nodes 4,6,7 are specified in the same multicast group, and the nodes 12, 15 are specified in the same multicast group, the nodes 24, 25 are specified in the same multicast group. The simulation results are shown in Figure 5.

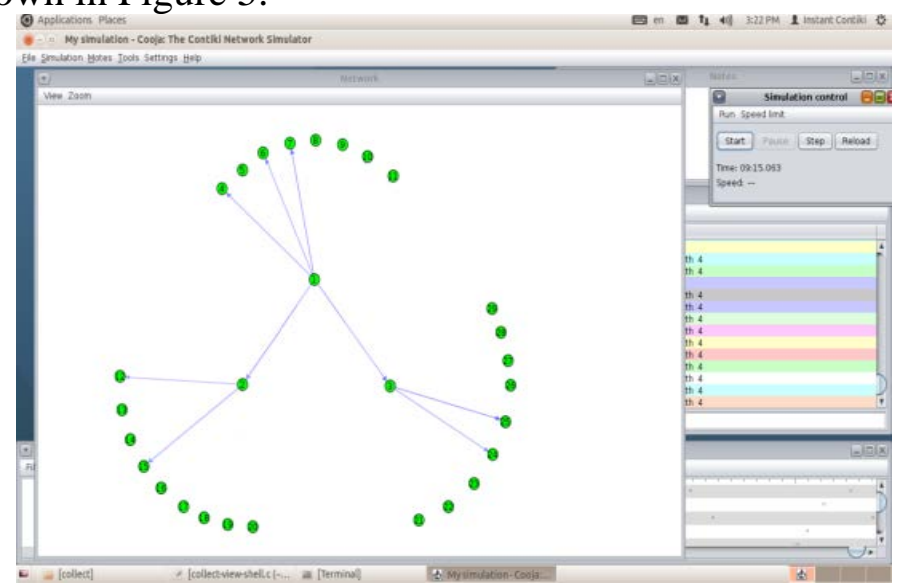

Fig. 5. The simulation of multicast

\section{The construction of system}

On the self-built platform, select a gateway, two routing nodes and 26 ordinary child nodes. As shown in Figure 6, we use national civil radio metering. The working frequency of metering is $470-$ $510 \mathrm{MHz}$. the underlying of the metering supports $802.15 .4 \mathrm{~g}$ standard.
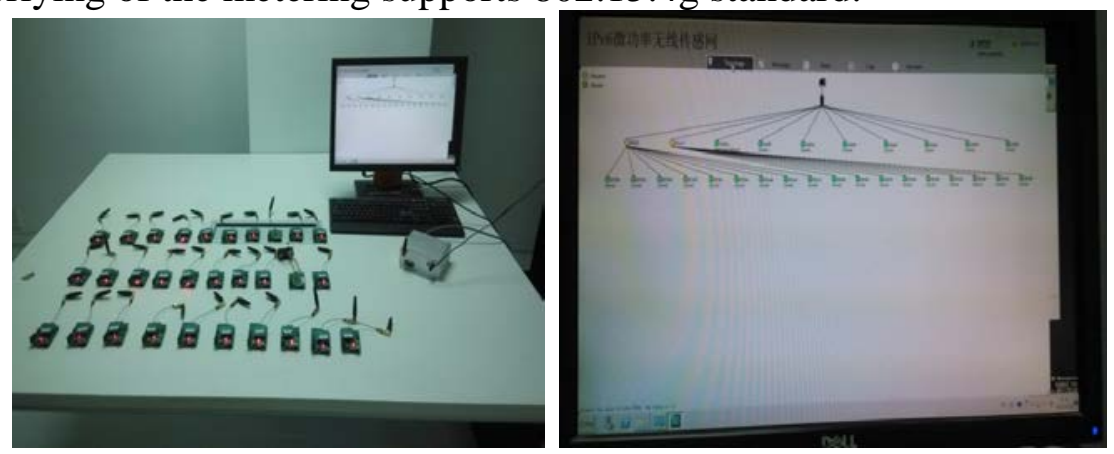

Fig. 6. platform schematic

\section{Conclusion}

This paper study the wireless sensor network protocol based on IPv6. Due to the shortage of existing broadcasters metering in the electricity information collection system, this paper proposed the multicast metering methods. And proposed increasing redundant tree routing in the basis of RPL protocol and proposed multicast trickle algorithms. This algorithm can collect the information of the designated multicast address. And simulate and implement the algorithm. Finally, we complete the 29-node system. 


\section{Acknowledgment}

When implementation of the systems, the signals from the wireless sensornetworks are preprocessed with the "SmartSignalProcessing" software developed by Prof. Ming-Yue ZHAI from North China Electric Power University.

\section{References}

[1] Bin Tian , Kun Mean Hou ,Hongling Shi ,Xing Liu. “Application of Modified RPL Under VANET-WSN Communication Architecture”,International Conference on Computational and Information Sciences (ICCIS), 2013 , Page(s):1467- 1470

[2] Korbi, I.E. “ility Enhanced RPL for Wireless Sensor Networks", international Conference on Network of the Future (NOF), Page(s):2012, 1- 8.

[3] Yang Zhi-jun , Yu Jin-cheng. "Research and implementation of IPv6 controllable multicast in the campus network”, International Conference on Wireless, Mobile and Multimedia Networks, 2010, Page(s):48- 50.

[4] YuanyuanYang. “A Service-Centric Multicast Architecture and Routing Protocol”, IEEE Transactions on Parallel and Distributed Systems, 2008, Page(s):35-51.

[5] Mukhtar, H. "Included in Your Digital SubscriptionLNMP-Management architecture for IPv6 based low-power wireless Personal Area Networks (6LoWPAN)", Network Operations and Management Symposium, 2008, Page(s): 417- 424.

[6] Yan-Hong ZHAO,Jie TONG, Ming-Yue ZHAI,Dan LI.Application of IPV6 wireless sensor network on advanced measuring system for smart grid. Applied Mechanics and Materials, $\mathrm{v}$ 602-605, p 2426-2430, 2014, Advanced Manufacturing and Information Engineering, Intelligent Instrumentation and Industry Development.

[7] XING Gui-Lan, ZHAI Ming-Yue, SUN Xiao-Da, "Self-similarity of Traffic in Wireless Sensor Networks for Smart Grid", IJACT: International Journal of Advancements in Computing Technology, Vol. 5, No. 8, pp. $524 \sim$ 531, 2013

[8] JIA SHu-Guang, LU Li-Peng, SU Ling-Dong, XING Gui-Lan, ZHAI Ming-Yue. An Efficient SleepingScheduling for Save Energy Consumption in Wireless Sensor Networks. Advanced Materials Research. Vol.756-759, p 2288-2293. 2013 